\title{
Femtosecond Stimulated Raman Study of Energy Transfer in Allophycocyanin
}

\author{
Sangwoon Yoon and Richard A. Mathies ${ }^{\dagger}$ \\ Department of Chemistry, Dankook University, Seoul 140-714, Korea. "E-mail: sangwoon@dankook.ac.kr \\ "Department of Chemistry, University of California, Berkeley, CA 94720, U.S.A. \\ Received July 18,2007
}

Key Words : Allophycocyanin, Phycobiliprotein, Femtosecond stimulated Raman, Energy transfer

Allophycocyanin (APC) is a light-harvesting protein found in cyanobacteria and red algae. ${ }^{1} \mathrm{APC}$ along with other phycobiliproteins such as phycocyanin (PC) and phycoerythrin (PE) constitutes phycobilisome and plays an important role in collecting light and cascading the excitation energy into a reaction center where the light energy is converted into chemical potential. ${ }^{2}$ Thus it is important to understand how excitation energy is transferred between the phycobiliproteins and inside them.

$\mathrm{APC}$ is a trimer of $\alpha \beta$ subunit, each of which contains a linear tetrapynole chromophore, phycocyanobilin (PCB), covalently bound to a cystein residue (Fig. 1). Close proximity $(\sim 20 \AA)^{3}$ between $\alpha-84$ and nearby $\beta-84$ in APC can cause the electronic states of the chromophores to interact to form split exciton states. In this case, interexciton dynamics mediates the energy transfer. ${ }^{45}$ On the other hand, it is also possible that energy transfer from $\alpha-84$ to $\beta-84$ occurs via dipole-dipole coupling between the two chromophores (Forster model) ${ }^{6.7}$ The intermediate range of distance between the PCBs in APC makes both models quite feasible for an energy transfer mechanism and has generated much controversy. Here we report on the excited state dynamics of APC measured by femtosecond stimulated Raman spectroscopy.

Femtosecond stimulated Raman spectroscopy (FSRS) reveals the structure of fast-evolving molecules by providing vibrational spectra with excellent spectral resolution on a femtosecond time scale. ${ }^{8-10}$ We prepare the electronic excited state of APC by $40 \mathrm{~nJ}, 30 \mathrm{fs}$ actinic pump pulse at 1 $\mathrm{kHz}$, centered at $610 \mathrm{~nm}$. The femtosecond stimulated Raman spectra of the excited state APC are obtained at a series of time delays by coupling $0.5 \mu \mathrm{J} /$ pulse, $2 \mathrm{ps}, 800 \mathrm{~nm}$ Raman pump pulse and $4 \mathrm{~nJ} /$ pulse, $20 \mathrm{fs}$, broadband continuum Raman probe pulse. The detailed description of the detection system and how to generate the laser pulses has
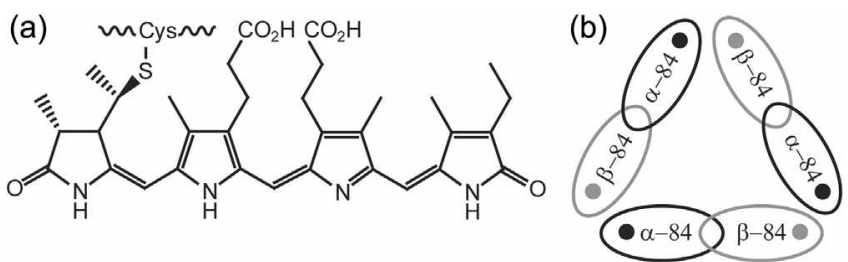

Figure 1. (a) Phycocyanobilin (PCB). (b) Schematic representation of $A$ PC. $a-84$ and $\beta-84$ indicate the PCBs linked to cystein- 84 in $a$ and $\beta$ subunits, respectively. been published elsewhere. ${ }^{11}$ APC was isolated from the filamentous cyanobacterium Anabaena variabilis ${ }^{12}$ and used in phosphate buffer solution.

Figure 2 presents the FSRS spectra of the ground state and the excited state APC at selected time delays. The excited state spectra were obtained by subtracting the spectra of buffer solution and the ground state APC which accounts for the unexcited $85 \%$ of APC. The ground state FSRS spectrum is in excellent agreement with the resonance Raman spectra previously reported at UV wavelengths. ${ }^{13}$ The excited state spectra show broad and dispersive features without any well-defined peaks. The amplitude of the dispersive features increases up to -80 fs and decreases gradually, completely gone by $5 \mathrm{ps}$ (not shown).

McCamant $e t$ al. ${ }^{14}$ found that dispersive lineshapes appear when the Raman pump and probe pulses drive resonant stimulated emission from the excited state and create vibrational coherence on the electronic ground state surface, which they termed the Raman initiated by nonlinear emission (RINE). The RINE spectra contain the information on the vibrational frequencies of the electronic gronnd state whose dynamics is determined by the evolution of the excited state population. From this model, McCamant $e t a l$. successfully extracted the vibrational spectra of the ground state bacteriorhodopsin, which evolve with the population changes in the excited state. ${ }^{14}$

Adopting a similar method described in ref. 14, we fit the excited state FSRS spectra of APC to the RINE model. From

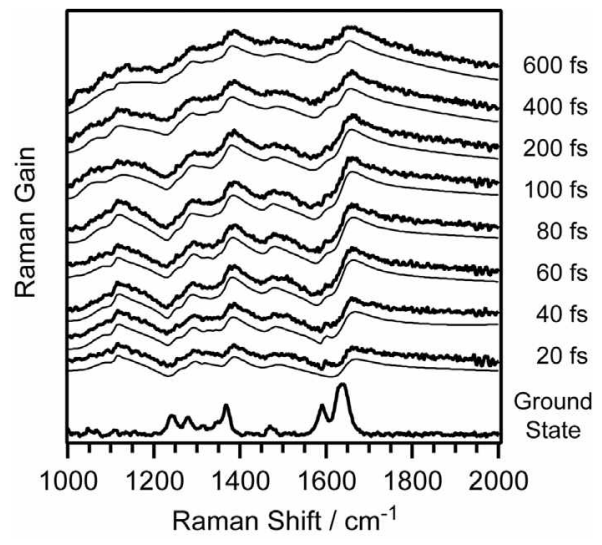

Figure 2. Femtosecond stimulated Raman spectra of excited APC at selected time delays. Thin lines are the best fit of the spectra to the RINE model described in the text. Spectra are offset for clarity. The FSRS spectrum of the ground state APC is also shown. 
(a)
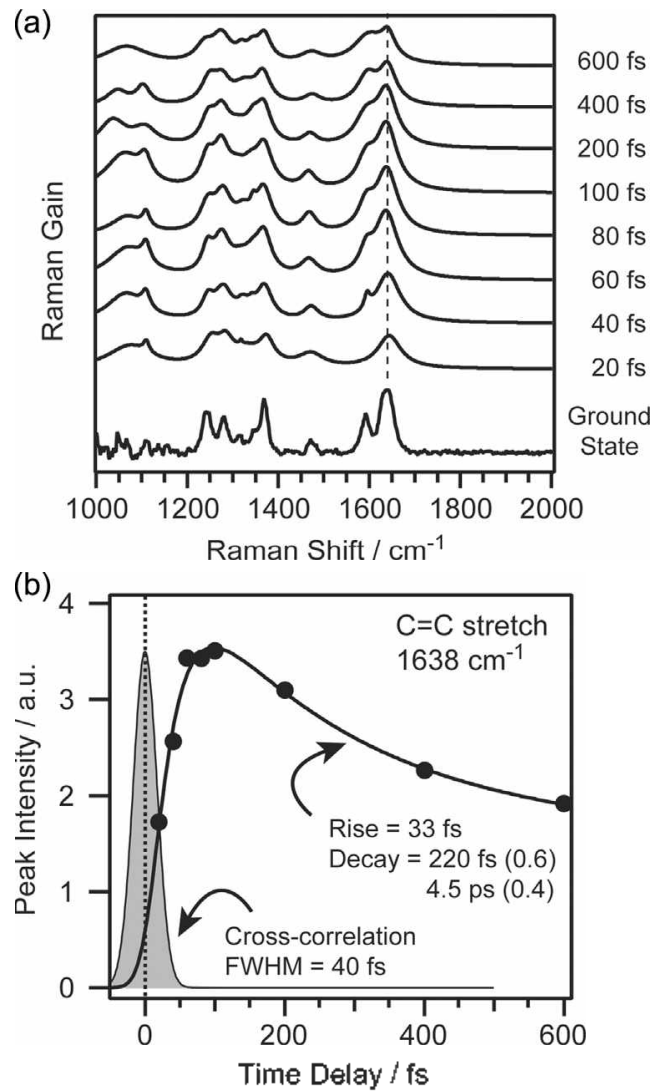

Figure 3. (a) Reconstructed Lorentzian lineshape spectra obtained by fitting the excited state FSRS spectra using the RINE model in Figure 2. Ground state spectrum is included for comparison. The dashed line indicates the $1638 \mathrm{~cm}^{-1} \mathrm{C}=\mathrm{C}$ stretching band. (b) Solid circles represent the amplitudes of the $1638 \mathrm{~cm}^{-1}$ band as a function of time delay. The solid line is the best fit to the experinental data with exponential functions, convoluted with the Gaussian instrument response function (FWHM-40 fs).

the best fit shown in Figure 2, we obtain ten ground state vibrational frequencies, amplitudes, and widths, from which normal Lorentzian lineshape vibrational spectra are reconstructed, as presented in Figure 3(a). The reconstructed Lorentzian spectra resemble the ground state spectra, confirming that RINE creates vibrational coherence in the ground state. The peak intensities of the reconstructed spectra are a direct measure of the population of the excited state at the given time delay. We plot the amplitude of the $1638 \mathrm{~cm}^{-1}$ band $(\mathrm{C}=\mathrm{C}$ stretch $)$ and $\mathrm{fit}$ it to a sum of exponential functions convoluted by a $40-\mathrm{fs}$ instrument response (Fig. 3(b)). The best fit yields three time constants: $33 \mathrm{fs}, 220 \mathrm{fs}$, and $4.5 \mathrm{ps}$. Due to the lack of data points at early and late time delays, however, 33 fs and $4.5 \mathrm{ps}$ have large uncertainties.

Our observations indicate that the population of the initially prepared excited state decays at the rate of $(220 \mathrm{fs})^{-1}$ to a state where the resonance condition for the stimulated emission at NIR is not met any more. The similar time scale has been observed in previous studies. ${ }^{47,15}$ One possible explanation is that the only $\alpha-84(\beta-84)$ is excited and the excitation energy is transferred to a nearby $\beta-84(\alpha-84)$ by the Forster energy transfer mechanism. However, it requires a premise that local protein environments are significantly different between the two chromophores and, as a result, the resonance condition is satisfied only for one chromophore. Another possible interpretation is that the initial excited state is a coupled exciton state and the excited exciton localizes onto one of the dimer chromophores with a time constant of $220 \mathrm{fs}$. Since the energy level of the exciton state is different from that of a decoupled local chromophore, the resonant stimulated emission condition (and thus the amplitudes of the reconstructed RINE spectra) changes over time as the localization of exciton occurs. ${ }^{3.15}$ Beck and coworkers reported that stimulated emission of APC in $700-750 \mathrm{~nm}$ shifts to the blue within $2 \mathrm{ps}$, supporting our interpretation. Thus, although further investigation is necessary, it appears that fast and efficient energy transfer in APC is mediated by the exciton localization.

In conclusion, we observed the excited state femtosecond stimulated Raman spectra of APC, dominated by broad dispersive lines. From the fitting of the spectra to the RINE model, we reconstructed the ground state vibrational spectra whose evolution is induced by the excited state dynamics. The vibrational intensities of the reconstructed spectra decay with a time constant of $220 \mathrm{fs}$, suggesting that the exciton localization occurs on this time scale and contributes to fast energy transfer in APC.

Acknowledgments. We thank Prof. A. N. Glazer for providing us with invaluable APC samples. We also thank Philipp Kukura for his help. This work was supported by the KRF gant (KRF-2006-331-C00141).

\section{References}

I. Glazer, A. N. Annu. Rev Biophys. Biophts. Chem. 1985, 14, 47.

2. Sauer, K. In Bioenergetics of Photosywhesis; Govindjee, Ed.; Academic Press: San Francisco, 1975; p 115.

3. Brejc, K.; Ficner, R.; Huber, R.; Sleinbacher, S. J. Mol, Biol. 1995 , 249.424.

4. Edington, M. D.; Riter, R. E.; Beck, W. F. J. Phss. Chem. 1995. $00,15699$.

5. Edinglon, M. D.; Riter, R. E.; Beck, W. F. J. Phys. Chem, 1996, 100,14206 .

6. Sharkov, A. V.; Kryukov, I. V.; Khoroshilov, E. V.; Kryukov, P. G.; Fischer, R.; Scheer, H.; Gillbro, T. Chem. Phys. Lett. 1992, 191, 633.

7. Xie, X.; Du, M.; Mets, L.; Fleming, G. R. SPIE 1992, $1640,690$.

8. Kukura, P.; McCamant, D. W.; Yoon, S.; Wandschneider, D.; Mathies, R. A. Science 2005, 310, 1006.

9. Lee, S.-Y.; Zhang, D.; McCamant, D. W; Kukura, P.; Mathies, R. A. J. Chem. Phys. 2004, J21, 3632.

10. Yoon, S.; Kukura, P.; Stuart C. M.; Mathies, R. A. Mol. Phys. 2006, 104,1275 .

11. McCamant, D. W; Kukura, P.; Yoon, S.; Mathies, R. A. Rev; Sci. Instrum, 2004, 75, 4971.

12. Bryant, D. A.; Glazer, A. N.; Eiserling, F, A. Arch. Microbiol. $1976,110,61$.

13. Szalontai, B.; Gombos, Z.; Csizmadia, V.; Csatorday, K.; Lutz, M. Biochemistry 1989, 28, 6467.

14. McCamant, D. W.; Kulura, P.; Mathies, R. A. J. Phss, Chem, B $2005,105,10449$.

15. Beck, W. F,; Sauer, K. J. Phss. Chem. 1992, 96, 4658. 